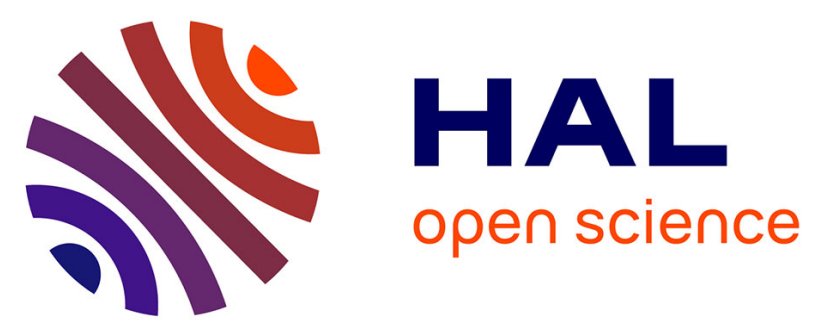

\title{
Vivre en zone contaminée ou les paradoxes de la gestion du risque
}

Guillaume Grandazzi, Yves Dupont, Laurent Bocéno, Frédérick Lemarchand

\section{To cite this version:}

Guillaume Grandazzi, Yves Dupont, Laurent Bocéno, Frédérick Lemarchand. Vivre en zone contaminée ou les paradoxes de la gestion du risque. Innovations et Sociétés, 2000, Connaissance et risque, 1, pp.41-64. hal-02125984

\section{HAL Id: hal-02125984 \\ https://hal-normandie-univ.archives-ouvertes.fr/hal-02125984}

Submitted on 10 May 2019

HAL is a multi-disciplinary open access archive for the deposit and dissemination of scientific research documents, whether they are published or not. The documents may come from teaching and research institutions in France or abroad, or from public or private research centers.
L'archive ouverte pluridisciplinaire HAL, est destinée au dépôt et à la diffusion de documents scientifiques de niveau recherche, publiés ou non, émanant des établissements d'enseignement et de recherche français ou étrangers, des laboratoires publics ou privés. 
Bocéno L., Dupont Y., Grandazzi G., Lemarchand F., « Vivre en zone contaminée ou les paradoxes de la gestion du risque ", Innovations et sociétés, 2000, n¹, p. 41-64.

\title{
Vivre en zone contaminée ou les paradoxes de la gestion du risque
}

\author{
L. BOCENO, \\ Y. DUPONT, \\ G. GRANDAZZI, \\ F. LEMARCHAND
}

\section{RÉSUME}

La catastrophe de Tchernobyl se présente à l'humanité comme une nouveauté radicale, tant par ses aspects technologiques que par les conséquences sociales qu'elle a entraînées. Loin de s'être résorbés ou encore d'avoir été liquidés comme il a été laissé entendre un peu rapidement, les effets des radiations et des contradictions qu'elles produisent, effets physiques, psychiques et sociaux, se font plus que jamais problématiques dans un monde où se sont effondrés simultanément deux grands « vecteurs » de la modernité que sont la science - le projet technico-scientifique et la confiance qui doit l'accompagner - et l'histoire l'utopie du communisme disparaissant avec le mur de Berlin. Restent donc des hommes, six millions au moins, plus que jamais démunis de repères tangibles, de mémoire collective et de références qui leur permettraient d'affronter pratiquement et symboliquement cette situation inédite : devoir (sur)vivre dans des territoires contaminés. La recherche menée par le LASAR ne vise rien moins qu'à tenter de comprendre, avec l'humilité qui s'impose, les logiques humaines à l'oeuvre dans la culture que doivent inventer les nouveaux cobayes de l'ère technologique pour assurer leur permanence au monde. C'est justement au travers de la parole des « sans-parole » de Tchernobyl, habitants, témoins, liquidateurs, que nous nous sommes efforcés de saisir, d'un point de vue sociologique et anthropologique, les modalités d'adaptation de la culture à cette situation, allant du déni et de la résignation à l'appropriation du risque.

\section{Que sait-on de Tchernobyl ?}

L'accident de Tchernobyl, sans précédent par l'ampleur, la nature et la durée de ses conséquences, pose le problème du devenir des populations habitant des territoires contaminés. Ainsi, dans le domaine des atteintes majeures à l'environnement affectant la santé des populations, la maîtrise de l'information et de la gestion des conséquences est loin d'être assurée. Les savoirs et les stratégies en la matière restent, depuis douze ans, relativement lacunaires et incertains et si nous avons tenu à aller arpenter les zones contaminées à hauteur d'homme, c'est que nous savions - du moins en avions-nous l'intuition que tout n'avait pas été dit sur cet événement que, par ailleurs, l'on s'empressait d'oublier, en particulier dans les pays fortement nucléarisés. Cette impression vague et intuitive que, finalement, nous ne savions pas grand 
chose de la catastrophe ni de ses conséquences ${ }^{1}$, non du point de vue des faits, mais de celui du sens, a également présidé à l'entreprise de l'écrivain et journaliste biélorusse Svetlana Alexiévitch, ainsi qu'elle s'en explique dans la préface de son ouvrage dédié aux victimes de Tchernobyl : "Dix années ont passé et Tchernobyl est devenu une métaphore, un symbole. Et même une histoire. Des dizaines de livres ont été écrits, des milliers de mètres de bandes-vidéo tournés. Il nous semble tout connaître sur Tchernobyl : les faits, les noms, les chiffres. Que peuton y ajouter? (...) ce livre ne parle pas de Tchernobyl mais du monde de Tchernobyl. Justement de ce dont nous connaissons peu. De ce que nous ne connaissons presque rien $»^{2}$. Plus, nous avions, comme l'auteur avec qui nous avons longuement échangé, l'impression grandissante que seule une parole, celle des vaincus, des victimes silencieuses et oubliées de cette histoire, les « sans-noms » de la catastrophe, pourrait nous aider à comprendre et donc à agir. Face à la nécessité, pour les habitants, de pouvoir nommer la chose, la catastrophe, comme à celle de donner un sens à l'inédit, nous avons choisi de procéder à une double approche à la fois sociologique et anthropologique, dans le but de procéder à une herméneutique des événements tout en produisant une historiographie à hauteur d'homme de ce passé qui semble refuser de passer. Procéder de cette historiographie signifie alors interroger d'un point de vue critique cette histoire-là, telle que la question a été formulée dans la préface précitée : «Une histoire manquée : voilà comment j'aurais pu l'intituler. L'événement en soi - ce qui s'est passé, qui est coupable, combien de tonnes de sable et de béton a-t-il fallu pour ériger le sarcophage au-dessus du trou du diable - ne m'intéressait pas. Je m'intéressais aux sensations, aux sentiments des individus qui ont touché à l'inconnu. Au mystère. Tchernobyl est ce mystère qu'il nous faut encore élucider $»^{3}$.

Nous pourrions donc procéder, comme il est usuel de le faire lorsqu'il s'agit d'appréhender quantitativement une catastrophe, à une énumération de chiffres vertigineux, traduisant l'ampleur du désastre, ses conséquences potentielles sur la santé de millions d'êtres humains à travers le monde. la durabilité des effets écologiques, économiques et sanitaires, ou le désarroi des politiques et gestionnaires soviétiques et occidentaux face à l'ampleur du désastre. L'on pourrait dès lors se perdre en confusion si l'on tentait obstinément d'analyser l'événement selon les logiques formelles en termes d'efficience des politiques d'intervention, de coûts-bénéfices, de gestion de crise ou encore de réparation. Bella et Roger Belbéoch ${ }^{4}$ avaient très tôt révélé l'inadaptation de la seule unité « rationnelle » dont nous disposons, l'argent, pour évaluer les composantes des préjudices que peut engendrer l'énergie nucléaire : « des experts travaillent sur ce problème pour tenter d'évaluer « rationnellement » le prix d'une vie humaine. Mais comment résoudre des équations du type : « enfant + argent $=$ enfant normal »? Cette

\footnotetext{
${ }^{1}$ Il est impossible, de ce point de vue, d'obtenir des données officielles fiables, pas plus que nous ne pouvons accorder de crédit aux chiffres des agences internationales qui ont, pendant dix ans, soutenu la thèse selon laquelle l'accident n'aurait produit « que » 32 morts (les ouvriers de la centrale). Nous avons constaté qu'environ un enfant sur deux, dans les écoles que nous avons visitées sur un mode aléatoire et sans prévenir, souffrait de problèmes de santé graves (affections thyrö̈diennes, problèmes cardio-vasculaires, ... ). Tous les habitants de la zone, et en particulier les enfants, souffrent, globalement, d'un affaiblissement de l'organisme et des défenses immunitaires. ${ }^{2}$ Alexiévitch S., La Supplication. Tchernobyl, chroniques du monde après l'apocalypse, Paris, Lattès, 1998.

${ }^{3}$ Ibid., p. 25.

${ }^{4}$ Roger et Bella Belbéoch, physiciens, furent à notre sens les premiers à livrer une véritable analyse, éclairée et fortement étayée, de la catastrophe de Tchernobyl dans leur ouvrage intitulé : Tchernobyl, une catastrophe, Allia, 1991.
} 
attitude « rationnelle » des promoteurs de l'industrie nucléaire conduit à considérer la vie des individus comme une simple valeur d'échange. Dans cette perspective, poursuivaient-ils, «il est parfaitement cohérent d'implanter des usines dangereuses et des centres de stockage de déchets dans les pays sous-développés où la valeur d'échange de la vie est très faible ». Il semblerait en effet que toute tentative de « rationalisation » de la catastrophe par les moyens techniquement disponibles, de l'économie, de la gestion ou même d'une macro-sociologie soit vouée à l'échec et réduite à l'impuissance. Chaque chiffre, chaque donnée quantitative supplémentaire avancée, si elle importe du point de vue de la restitution de la dimension réelle insoupçonnée et souvent dissimulée encore de la catastrophe, ne facilite par pour autant la compréhension de l'événement qui semble refuser obstinément de s'inscrire dans l'histoire. C'est pourquoi nous avons envisagé, il y a trois ans, de nous lancer en quête de la parole des « sans parole », des survivants du «monde nouveau » issu de la première catastrophe nucléaire civile.

\section{Analyses empiriques}

Nous nous proposons de procéder, à l'issue d'une première série d'observations empiriques, à une catégorisation analytique de la manière dont les individus condamnés à devoir - essayer - d'habiter un vaste territoire contaminé construisent un rapport avant tout social et symbolique à la contamination, au danger, à l'espace et au temps, dans la nécessité de concilier les très nombreuses contradictions inhérentes à leur situation. La mémoire de l'accident et de ses conséquences catastrophiques interfère, d'abord, avec celle de la seconde guerre mondiale, dont la Biélorussie fut le théâtre des pires atrocités, du front russe à la Shoah. Mais la durée des effets, agissant comme une «bombe à retardement», empêche en même temps le rapprochement des deux événements dans les mémoires : il faut donc, faute d'expérience, se résoudre à penser Tchernobyl comme quelque chose de radicalement nouveau, d'inédit. Contrairement aux sutures des blessures de guerres, l'oubli et donc le deuil de Tchernobyl semblent d'autant plus difficiles à réaliser que de nouvelles cohortes de malades et de décès viennent sans cesse réactiver la mémoire de l'accident et rappeler à ceux qui y ont survécu que les effets apparaissent toujours, et se multiplient. La population semble ainsi prise dans une contradiction première, entre un désir d'oublier et un besoin de se souvenir, l'exercice de l'un et de l'autre étant finalement rendus impossibles. La catastrophe a, ensuite, à de nombreux égards, constitué individuellement et collectivement, une rupture importante, au moins aussi importante que la seconde guerre mondiale, en tant qu'elle permet de définir un " avant » et un « après ». Mais elle n'est pas tant liée, pour les anciens, et principalement les kolkhoziens, à la présence d'une contamination dont ils ne reconnaissent pas toujours l'existence, qu'à l'abandon des villages et des lieux de vie jadis animés. Le départ des jeunes, bien souvent précipité avec la catastrophe, constitue l'un de leurs principaux regrets et en ce sens, Tchernobyl a précipité la dynamique sociale d'éloignement des générations qui s'est enclenchée avec l'exode rural, le désir d'urbanité de la jeune génération, la mécanisation agricole et la diminution des actifs agricoles dans les campagnes des pays de l'ex-URSS. 
La confusion des effets des deux phases, accidentelle et post-accidentelle, de l'accident constitue en fait un puissant facteur d'anxiété, cette confusion entraînant une situation paradoxale, un effet d'après-coup, que les habitants traduisent par l'expression : " la catastrophe est un arbre qui pousse ». Nous allons tenter de mettre en place les éléments qui nous permettront d'engager une réflexion sur l'idée de rupture, en particulier de l'univers de représentations de l'espace et du temps. Nous nous devons donc d'être attentifs autant à la "gestion des risques ", aux différentes manières qu'ont les individus de faire face, et bien souvent de « faire avec » la contamination, qu'à la façon dont celle-ci a pu finalement modifier les repères spatiaux et temporels habituels.

La longue enquête à laquelle nous nous sommes livrés nous aura permis de mesurer, dans un premier temps, les effets de la mise en oeuvre de mesures de protection radiologique, mesures qui peuvent paradoxalement contribuer à renforcer l'inquiétude de la population en tant qu'elles signifient l'existence d'un risque et la nécessité de s'en protéger. La confusion entre les deux phases, outre le fait qu'elle est un facteur d'anxiété de la population, a des effets paradoxaux : elle peut amener les individus à se focaliser sur la contamination et conduire à une perte de dynamisme social (paralysie), voire à des syndromes de dépression collective ; elle peut aussi contribuer à un remaniement psychique des représentations associées à la contamination et au risque qui en découle. Ainsi, la perception du risque lié à la contamination s'estompe avec le temps, le déni du risque permettant aux individus de juguler le stress lié à la vie en zone contaminée. Nous entendions dire par exemple : "l'organisme s'habitue ", "le corps devient plus résistant", "la contamination n'est plus dangereuse après un certain temps », " comme les doryphores avec les pesticides, les hommes s'habituent aux radiations ». Dans la situation d'impuissance où est plongée la population vivant dans les territoires contaminés, nous avons relevé deux attitudes principales permettant de faire face au risque : la résignation et le déni. Quand le risque est pris en compte par les individus, l'impossibilité pratique d'avoir prise sur celui-ci (impossibilité de déménager, absence de moyens d'action sur place... ) provoque un stress important, d'où une perte de dynamisme qui engendre le plus souvent un fatalisme. A l'inverse, la diminution du stress ne semble possible qu'au prix d'un déni du risque, rendu possible par un remaniement des représentations et des constructions sociales. Entre ces deux types d'attitudes les plus répandus, un certain nombre de personnes oscille d'un registre à l'autre, leur discours révélant les contradictions dans lesquelles elles sont prises, que seuls des entretiens approfondis permettent de mettre en évidence.

L'une des principales conséquences de la catastrophe, liée aux effets de la gestion qui en a été faite, et des plus importantes à notre sens, est la perte de confiance de la population dans le discours des autorités. Cette défiance apparaissait comme étant particulièrement marquée vis-à-vis des autorités, mais aussi vis-à-vis de ceux qui sont considérés comme ayant profité du dispositif de gestion post-accidentel : ceux qui ont reçu un second appartement dans une autre ville, les spécialistes qui touchent un double salaire pour travailler en territoire contaminé, les responsables de kolkhoze qui exploitent des terres agricoles interdites à la production, etc. Les responsables du contrôle sanitaire ne semblaient pas, par ailleurs, être réellement indépendants des autorités locales, la crédibilité du dispositif de contrôle s'en trouvant sérieusement mise en doute. Toutefois, si beaucoup ont repris leurs « habitudes » de 
vie, ceci n'est vrai qu'en apparence dans la mesure où l'on ne peut pas objectivement parler de retour à une « situation normale » pour ces populations. Il est d'ailleurs frappant de constater combien en première approximation, la vie semble routinière et s'écouler « normalement » dans les territoires contaminés. A ce niveau, différents facteurs, qu'il s'agirait d'affiner, contribuent à empêcher un processus social de reconstruction post-accidentelle comme c'est souvent le cas après une catastrophe naturelle ou une guerre. Cela signifie explicitement que les populations ne peuvent mobiliser qu'une culture ancienne et anachronique de la catastrophe pour faire face à l'inédit, au radicalement nouveau.

Le retour à la vie normale n'est donc pas sans poser problème sur le plan de la protection radiologique dans la mesure où une population qui a pris son parti de rester sur les territoires contaminés peut être amenée à minimiser et à banaliser le risque radiologique. Ainsi des observations statistiques ont permis de mettre en évidence en $1994^{5}$, que l'on pouvait assister dans certaines régions contaminées de Russie, à une remontée des doses individuelles de contamination, ce qui semble confirmer le changement de comportement d'une population vivant un retour progressif à une vie normale et reprenant peu à peu ses habitudes (pêche, cueillette de champignons, culture du potager) au préjudice de son niveau de protection radiologique. Or, il apparaît aujourd'hui qu'aucun espoir de mieux-être n'accompagne plus l'effort demandé, en terme d'adaptation, aux populations concernées. Cette question est relayée par l'effondrement brutal du système politico-économique qui pouvait apporter - entre deux famines - dans les pays communistes, un minimum de garanties aux personnes (droit au logement, alimentation). Par ailleurs, la désillusion qui accompagne les promesses non tenues des bienfaits du «libéralisme » naissant, dont la manifestation la plus visible n'a été pour beaucoup que l'économie totalement déréglementée de la mafia, renforce ce phénomène de repli sur les conditions de vie, sociales et économiques, qui ont précédé l'accident.

La vie en territoire contaminé, entre gestion du risque et retour à la vie normale : deux tendances contradictoires.

L'analyse des entretiens réalisés auprès de la population des districts de Kostiokovitchi et de Krasnapolié ${ }^{6}$ révèle la contradiction profonde et l'ambivalence attachées à la vie dans les territoires contaminés. En effet, la présence durable de la contamination radioactive dans une large partie des districts confronte les habitants à un risque appelé « résiduel » et persistant. Ainsi, dans les zones les plus contaminées, l'absence de mesures de radioprotection entraîne une exposition des résidants supérieure à celle définie pour le public par les normes internationales en situation non accidentelle. Dans ces conditions, et compte tenu de l'incertitude qui caractérise l'évaluation des effets stochastiques ${ }^{7}$ liés aux faibles doses, des

\footnotetext{
${ }^{5}$ Communication de J.-P. Revel, lors du colloque «2 d international scientific conférence on consequences of Chemobyl catastrophe », Genève, 13 et 14 novembre 1997 - Centre d'Ecologie Humaine, Université de Genève.

${ }^{6}$ Voir carte à la fin de l'article.

${ }^{7}$ Ce terme nécessite une précision selon nous importante. Il faut en effet distinguer nettement les effets dits déterministes (symptômes cliniques et biologiques d'une irradiation aiguë) que l'on connaît assez bien et que l'on peut identifier, des effets, liés aux faibles doses, dits non-déterministes, ou stochastiques. Ces derniers,
} 
mesures de protection radiologique s'imposent. Dans zones moins contaminées où l'exposition estimée est inférieure aux normes internationales, des actions de réduction du risque sont toutefois envisagées par les autorités et ces territoires font l'objet d'un « contrôle radiologique strict ». De plus, le caractère permanent de la contamination ainsi que la difficulté éprouvée à identifier précisément ses effets sur la santé contribuent à atténuer la vigilance de personnes qui disent s'être habituées, sinon «physiquement », du moins psychologiquement, en raison même de la durabilité de la situation radiologique due à l'accident. Encore une fois, cette « accoutumance » à la contamination ne doit pas laisser penser que ces personnes se sont habituées aux contre-mesures et qu'elles ont pris leur parti des nombreuses interdictions ou limitations qui pèsent encore maintenant sur la vie en zone contaminée, Elle a tendance au contraire à favoriser le développement de comportements qui ne relèvent pas d'une attitude précautionneuse, la contamination n'étant plus forcément l'objet d'une attention toujours renouvelée. Le discours des autorités s'alimente à certains égards de cet «état de fait» et légitime ainsi, non sans une pointe de fatalisme, sa politique de banalisation de la situation. L'impossibilité de désigner des responsables et une raison du malheur, le désir de retour à la normale ont conduit de très nombreux villageois à rendre finalement le relogement - et les autorités qui s'y sont attachées - seuls responsables de leur malheur. Le problème ne tient alors plus tant dans la contamination, dont l'existence est parfois mise en doute, que la ruinification des villages abandonnés qui se présente comme le signe de la fin d'un monde.

$\mathrm{Au}$ niveau politique, il existe indéniablement une volonté de réhabilitation des territoires contaminés qui rencontre, par affinité, le désir d'une partie de la population de vivre « normalement », comme avant. Cette volonté politique, en ce qu'elle vise essentiellement le redéploiement de l'activité économique et le redémarrage de la production agricole, conduit à une relativisation de la radioprotection qui peut s'avérer dommageable à long terme. Plus de treize ans se sont désormais écoulés depuis l'accident, huit depuis les premiers programmes nationaux mis en place pour indemniser et protéger les personnes vivant dans les territoires affectés par l'accident, et cette période, dotée d'une temporalité propre, semble à certains une phase de «transition » suffisamment longue d'efforts et de sacrifices consentis pour retrouver aujourd'hui des conditions de vie moins contraignantes sur le plan de la protection radiologique. Aussi, le retour à la normale s'apparente-t-il dans certains cas à l'absence de mesures visant à réduire le risque.

Mais le retour à la normalité, souhaité à la fois par les autorités et les habitants, apparaît à son tour comme un objectif préjudiciable à la gestion du risque radiologique, du fait de la persistance de la contamination post-accidentelle, au regard de laquelle une dizaine d'années semble bien peu. Il ne peut, et ne doit, s'agir que d'un objectif à très long terme, la confrontation durable de la population au risque résiduel n'autorisant pas un oubli progressif de la contamination qui serait plus rapide que la désintégration - lente mais réelle - des radioéléments. Aussi doit-on pointer les limites inhérentes à un dispositif de gestion post-accidentelle animé

aléatoires, nécessitent le recours à l'épidémiologie pour être mesurés statistiquement sur une population. Comme le soulignent $R$. et B. Belbéoch (note, op. cit.), ces derniers sont aussi qualifiés d'incertains, ce qui constitue une escroquerie linguistique : ils sont tout aussi certains que les effets déterministes, mais il ne peuvent être appréhendés, nous l'avons dit, directement par la médecine. 
par ce type d'intention, par ailleurs incompatible avec le caractère irréparable et irréversible que revêt pour les populations touchées, la situation post-catastrophique qui les contraint à vivre dans un environnement irrémédiablement dégradé.

Reprenons les résultats des analyses socio-anthropologiques selon lesquels deux tendances fondamentales et contradictoires structurent la vie en territoire contaminé. La première est signifiée dans un lexique "gestion du risque, vigilance, méfiance, précaution, etc. ", la seconde renvoie à l'aspiration évoquée plus haut d'un retour à la normalité. Les personnes interrogées ne se répartissent pas simplement entre celles qui, conscientes de la contamination et des risques encourus, auraient adopté des comportements « rationnels » de réduction du risque et les autres qui, pour des raisons diverses, auraient cessé d'être vigilantes, faisant désormais comme si de rien n'était. La réalité post-catastrophique est bien évidemment beaucoup plus complexe et chacun des entretiens effectués illustre à sa manière la contradiction dans laquelle sont pris les habitants dont les discours tiennent à la fois et dans des proportions diverses, de l'un et l'autre des registres. Cette contradiction est, en l'état actuel des choses, insurmontable dans la mesure où l'objectif de retour à la normale est à court terme irréalisable et conduit à un constat d'impuissance, lequel engendre tour à tour désespoir ou déni du risque. Plutôt que de chercher à sérier les différents types d'attitudes et représentations suscitées par la confrontation au risque, il a été jugé préférable de dégager ce qui, dans les propos des interviewés, était susceptible de favoriser des comportements adaptés aux exigences de la gestion du risque radiologique d'une part, et les pratiques et représentations qui semblent s'opposer à l'adoption d'une attitude inspirée par le principe de précaution d'autre part. Chaque situation individuelle constitue un cas particulier par rapport au risque et l'approche proposée ici entend esquisser une typologie, au sens weberien du terme, tendue entre ces deux pôles. La frontière entre les différents types d'attitudes face au risque dont nous allons donner une première ébauche est nécessairement floue et procède d'une démarche essentiellement méthodologique, aucun des types ainsi définis n'existant, comme nous l'avons analysé, «à l'état pur ». La construction idéal-typique doit néanmoins permettre de cerner la rationalité « dominante » chez telle ou telle personne, à partir de l'appréhension d'un rapport global à la menace dans le cadre particulier de la vie en territoire contaminé et à proximité.

\section{Première tendance : la prise en compte du risque radiologique}

Si les pratiques de radioprotection sont peu mises en oeuvre par les habitants et n'ont pu être directement observées lors des enquêtes de terrain, les investigations menées ont toutefois permis de recueillir des discours sur les pratiques qui témoignent de l'état des connaissances de la population en matière de radioprotection, et plus largement d'une culture du risque, de type rationnel, indépendamment de la mise en pratique de ces connaissances. D'une manière générale, les personnes disposant d'une culture scientifique et ne cautionnant pas la conception étatique actuelle de la "réhabilitation » des zones contaminées, placent leurs espoirs, fussent-ils modestes, dans la transmission d'une culture technique aux populations susceptibles de la recevoir, c'est-à-dire les enfants. Le contrôle radiologique de l'alimentation et de l'environnement constitue l'un des éléments du dispositif de gestion du risque mis en place au niveau individuel. Toutefois, le peu de confiance envers les contrôles officiels, mais surtout 
la récession économique n'encouragent pas les habitants à recourir aux services des laboratoires de contrôle radiologique. Jusqu'à Minsk, l'attitude face au contrôle atteste d'une confiance modérée et en même temps nécessaire dans le dispositif étatique de mesures radiologiques. Les autres mesures de réduction du risque évoquées lors des entretiens concernent pour l'essentiel la préparation des aliments, notamment les champignons et les produits de la chasse particulièrement contaminés, et ce pour longtemps, même en dehors des zones contrôlées. Ces mesures sont généralement ressenties comme des prescriptions négatives auxquelles il n'est pas toujours facile de se conformer, et ce d'autant plus qu'elles consistent d'ordinaire à se priver d'aliments appréciés et traditionnellement consommés (champignons, baies) ou d'activités plutôt agréables auxquelles permettrait de s'adonner la période estivale (baignades en rivière, promenades en forêt, etc.). Pour beaucoup, la capacité d'action individuelle sur le risque semble très réduite et s'apparente toujours à des restrictions qu'il faut s'imposer, à soi-même et aux enfants, ce qui donne lieu à une forme contradictoire de résignation que nous pourrions nommer comportement rationnel résigné. Le fait de ne pas se rendre en zones contaminées constitue, pour certains habitants des zones propres, la seule démarche possible pour se protéger de la contamination, ce qui n'est pas sans conséquences sur la pérennité des relations familiales et des liens inter-générationnels dès lors que les membres les plus âgés de la famille ont refusé d'être relogés et continuent à vivre en zone contaminée.

\section{Seconde tendance : l'aspiration au retour à la normale}

La volonté politique de « réhabilitation » des territoires qui devaient initialement être évacués, c'est-à-dire les zones enregistrant une activité surfacique de plus de $15 \mathrm{Ci} / \mathrm{km}^{2}(>555$ $\mathrm{kBq} / \mathrm{m}^{2}$ ), souffre d'un certain nombre d'ambiguïtés et de paradoxes, notamment quant à la compatibilité entre la reprise d'une activité économique et les contraintes de radioprotection. La politique actuelle de repeuplement des territoires contaminés, notamment en Biélorussie, par le recours à des mesures pour une part incitatives et, pour une autre, autoritaires, pourrait conduire à minimiser les incidences sanitaires de l'accident à long terme, et compromettre du même coup les conditions d'un retour à une vie normale qui ne soit pas le produit d'une banalisation - criminelle - du risque de contamination. La volonté d'un retour à la normale fait donc écho, au niveau collectif, au projet politique de réhabilitation des territoires contaminés, au moins jusqu'à $555 \mathrm{KBq} / \mathrm{m}^{2}$ (dégel des terres, mesures de relogement stoppées, encouragements à rester ou revenir vivre dans ces zones, etc.); au niveau individuel ou familial, elle se traduit d'une manière générale par une régression des pratiques de radioprotection mises en oeuvre par les habitants. Vivre comme avant l'accident revient ainsi pour beaucoup à ne prendre aucune mesure pour réduire le risque lié à la contamination, à faire comme si de rien n'était : attitude que nous nommerons dénégationniste. Le déni peut porter sur la réalité de la contamination, sur la réalité du danger et/ou sur les conséquences sanitaires qui lui sont associées, toutes formes de dénégation qui visent le plus souvent à fournir des explications rationalisées à un choix initial (refus du relogement par exemple) ou qui constituent un argumentaire étayé à partir du sentiment d'impuissance et «nécessaire » pour vivre dans des conditions de confort moral acceptable. L'impuissance ressentie, lorsqu'elle n'engendre pas la 
dénégation, conduit certains à la résignation sous la forme du fatalisme ${ }^{8}$. La contamination et le risque deviennent alors des réalités subies et sont évacuées des préoccupations quotidiennes. Certaines personnes se montrent ainsi désespérées et c'est en termes de "survie » qu'elles parlent de leur situation. C'est par le recours à la notion d'« habitude », polysémique chez les interlocuteurs, qu'ils signifient qu'ils se sont résignés à vivre avec la contamination, adversité devenue peu à peu présence indifférente, mais dont les effets délétères ne sont pas occultés.

Pour plusieurs médecins que nous avons rencontrés, la population est responsable de sa santé et ils peuvent parfois passer facilement du constat d'une diminution des mesures individuelles de réduction du risque, à une culpabilisation des familles pour lesquelles la contamination ne constitue plus une préoccupation centrale et qui ne respectent pas les interdictions ou leurs recommandations. Bella et Roger Belbéoch avaient bien pointé la perversité d'une telle politique, quand ils s'interrogeaient sur les raisons pour lesquelles les autorités s'acharnaient à maintenir un système d'interdictions alors que la population semblait se satisfaire d'une banalisation de la situation : "La responsabilité des autorités, en cas de dégradation de la situation, ne pourrait ainsi être mise en avant, seul le non respect des règles qu'elles avaient recommandées serait la cause des dégâts sanitaires. La population serait, enfin de compte, responsable des maux qu'elle devrait subir ${ }^{9}$. Les difficultés économiques rencontrées par les familles ont aussi fortement contribué à ce que la contamination ne soit plus leur préoccupation centrale faisant l'objet d'un souci permanent. Pour nombre d'entre elles, la baisse de la vigilance et des précautions ne résulte pas d'un choix mais bien d'une impérieuse nécessité économique qui ne leur laisse pas la possibilité d'agir autrement. Il ne s'agit pas d'acheter des produits propres, mais d'acheter des denrées alimentaires pour survivre. Pour certaines personnes interrogées, le moindre souci dont fait l'objet la contamination est assimilé à un retour à la raison, par opposition à la « panique » injustifiée de la population lorsqu'elle a commencé à bénéficier d'une information, qualifiée de « propagande ».

Ce sont, dans ce cas, les contre-mesures elles-mêmes qui sont considérées comme la source de tous les maux, ce discours étant repris par les autorités : le mieux, selon elles, aurait été de continuer à ne rien dire pour ne pas affoler « inutilement» les gens. Notons que ce type de discours caractérisé par un déni important du danger est essentiellement tenu par les habitants des zones très contaminées qui ont choisi de rester, choix qui entraîne généralement une modification des représentations concernant le risque. Le retour à la normalité et la reprise des habitudes de vie peuvent également résulter d'un sentiment d'impuissance face au danger. L'impression, voire la conviction, d'être incapable d'agir à titre individuel pour réduire le risque est en partie liée au caractère centralisé de la gestion post-accidentelle qui incite certaines

\footnotetext{
${ }^{8}$ E. Durkheim a mobilisé la catégorie explicative du fatalisme dans son étude du suicide en liant celle-ci à un excès de régulation. Durkheim E., Le Suicide, Paris, PUF Quadrige, 1986. Un monde surdéterminé par l'excès de contrainte, ici l'environnement contaminé, lié à l'impossibilité objective d'y échapper et à l'absence d'alternative, pourrait s'inscrire dans une telle catégorie. C'est dans ce sens que nous comprenons le fatalisme et non comme un supposé trait fondamental de la « culture » slave.

${ }^{9}$ Belbéoch R. et B., op. cit. p. 182.
} 
personnes à s'en remettre aux autorités compétentes, seules susceptibles à leurs yeux de pouvoir faire quelque chose. Nous avons, dans ce cas, affaire à une résignation confiante.

À l'inverse, l'incapacité des responsables politiques et des scientifiques à « liquider» les conséquences de l'accident peut aussi laisser penser que les mesures de protection individuelle sont inutiles, ce qui donnera lieu à une attitude de type désespéré.

\begin{tabular}{|c|c|c|}
\hline \multirow{3}{*}{ Actif } & $\begin{array}{c}\text { Prise en compte } \\
\text { du risque }\end{array}$ & Déni du risque \\
& $\begin{array}{c}\text { Attitude préventive } \\
\text { rationnelle } \\
\text { (fuir la zone ou } \\
\text { s'adapter) }\end{array}$ & Dénégation active \\
& (construit un récit de \\
& & l'absence de \\
& Rationnel résigné & contamination \\
& ou de danger; retour \\
& en zones) \\
& (fatalisme) & Résignation \\
& a plus riespéré (il n'y & confiante ou \\
& faire) & désespérée (les \\
& autorités \\
& arrangeront tout, ou \\
& pas, \\
& mais le problème \\
& n'est pas \\
& lié à Tchernobyl) \\
\hline
\end{tabular}

Tableau de synthèse de la typologie

En conclusion, à l'opposé du comportement de type rationnel, parfois même rationnel résigné, deux types d'attitudes sont engendrés par le constat d'impuissance lié à l'impossible réalisation de l'objectif d'un retour à la situation qui précédait l'accident. Le premier se caractérise par des formes diverses de dénégation, le second par les deux formes de la résignation confiante et du désespoir. A l'axe prise en compte/déni du risque correspond un jeu d'opposition des comportements selon les critères actif/passif qui permet de construire quatre types abstraits, et néanmoins validés empiriquement, d'attitudes face à la contamination. La 
construction typologique quadrilogique ${ }^{10}$ résultat de cette double tension de types mixtes (interdépendants) est résumée dans le tableau ci-dessus. Notons que la dénégation entre dans la catégorie de l'action dans la mesure où, comme nous l'avons déjà largement illustré, elle nécessite la construction de la preuve de l'absence de risque ou même de danger, tant par la production d'un récit que par la production et la mobilisation de preuves (allant jusqu'à la mesure physique).

\section{Approches théoriques}

\section{L'espace déstructuré.}

Avec la contamination radioactive, il faudrait inventer une nouvelle culture, intégrant le temps long et l'urgence, la contamination planétaire et ses variations les plus « locales ». Nous pouvons plus largement nous demander dans quelle mesure la catastrophe de Tchernobyl, qui présente à bien des égards une structure épidémique ${ }^{11}$, a pu engendrer une modification de nos rapports anciens à l'espace, au sens du territoire habité et garanti par des frontières. Inédite, elle se présente résolument comme un défi lancé à notre culture, moderne, de l'espace. A l'examen de la situation, des effets sociaux et culturels que nous avons étudiés au sein des populations biélorusses vivant dans les territoires fortement contaminés et éloignés de la centrale en tant que telle, il apparaît que chez un grand nombre de personnes, le système de représentations de l'espace et du temps par l'intermédiaire duquel elles avaient jusqu'à présent appréhendé le monde, à hauteur d'homme, a été profondément bouleversé. Ainsi, du point de vue de l'espace, de nombreuses difficultés, dues à la structure épidémique de la catastrophe, rendent impossible la construction d'une représentation objective de la situation. En premier lieu, la radiation est indécelable, inodore et sans saveur - du moins à présent - et, en tant qu'elle échappe à tous nos sens, elle ne peut donc être que symbolisée ou signifiée, c'est-à-dire rendue présente comme présence invisible. De ce point de vue, toute expérience de la vie en zone contaminée est déjà rendue a priori problématique, dans la mesure où « nous n'avons ni système de représentation, ni analogie, ni expérience (...) un événement auquel ne sont adaptés ni nos yeux, ni nos oreilles ni même notre vocabulaire " ${ }^{12}$. Seul un trop coûteux et rarissime appareillage de mesure, d'utilisation compliquée et d'efficacité limitée ${ }^{13}$, permettrait de déceler sa présence. Les cartes en vigueur ne fournissent que des données globales sur les grandes « zones » de contamination, en se bornant à quelques prescriptions et interdictions alimentaires, alors que les radionucléides sont en fait répandus sur le territoire en dessinant une multitude de taches, à la manière d'une « peau de léopard », dont les «points chauds » peuvent ne mesurer que quelques mètres carrés, localisés sous une évacuation de gouttière, dans une cour d'école, un jardin, etc. Dès lors, toute frontière appréhendée in situ, et non plus sur la carte, tombe dans

\footnotetext{
${ }^{10}$ Voir S. Juan, Méthodes de recherche en sciences sociohumaines, Paris, PUF, 1999, pp. 262-264.

${ }^{11}$ Voir Rieusset-Lemarié I., Une Fin de siècle épidémique, Actes Sud, 1992.

${ }^{12}$ Alexiévitch S., La Supplication, op. cit. p. 33.

${ }^{13}$ Les appareils les plus couramment utilisés ne relèvent que le niveau de radiation gammamétrique, c'est-à-dire principalement la présence de Cs137. Les virulents émetteurs alpha et bêta, présents dans l'alimentation, nécessitent, pour être détectés, des appareils de comptage trop coûteux.
} 
le domaine de l'absurde, une absurdité qui n'échappe pas aux habitants, comme l'a fait remarquer Yarochinskaya : "Les champs sont partagés par un ruisseau. D'un côté, on paie un complément, de l'autre non. Et pourtant la poussière, elle ne voit pas la différence, elle va des deux côtés ${ }^{14}$. La carte, comme fiction cartographique, diffusée par voie de presse, demeure pourtant le seul support par l'intermédiaire duquel les autorités transmettent aux populations une information - réduite au minimum - qui est censée être la réalité, mais qui n'entraîne que rarement, comme nous l'avons constaté, les comportements de radioprotection attendus. Ainsi se construit la fiction «officielle» d'un retour à la normale, via la carte de la supposée - contamination en $2016^{15}$.

Pire, la frontière de la zone contaminée agit parfois comme celle d'un asile (asilium, temple), à l'intérieur duquel on finit par se sentir à l'abri de la menace. Non seulement parce que la catastrophe est déjà arrivée, mais parce qu'aussi les repères, les valeurs qui affectaient les choses, les êtres, la nature, n'y sont pas totalement effondrés, mais plutôt brouillés. Ce fait, qui reste encore une fois selon nous impensé, est assez bien illustré par l'attitude ambiguë des chercheurs et écologistes qui accompagnèrent le cinéaste biélorusse Y. Chatchévatski, dans le tournage de son documentaire L'Oasis ${ }^{16}$, consacré à la vie d'un homme dans zone d'évacuation « totale » des trente kilomètres autour de la centrale, où l'on entend finalement ces voyageurs témoigner, non sans une certaine gêne, d'un étrange « sentiment de liberté » paradoxal. Mieux, le gouvernement biélorusse a mis en oeuvre, depuis le début des années quatre-vingt-dix ${ }^{17}$, une politique de création de parcs naturels nationaux, des « réserves de nature sauvage » où sont réintroduits, sous le contrôle attentif de l'Académie des Sciences, les traditionnels bisons (zubres) d'Europe centrale dans les zones les plus contaminées du pays, qui ont été (théoriquement) vidées de la totalité de leur population.

La catastrophe de Tchernobyl a également engendré, dans les pays d'Europe occidentale moins fortement touchés par la radiation, des modifications culturelles dont on sous-estime aujourd'hui probablement l'ampleur et que nous pourrions aborder au travers de deux exemples. Souvenons-nous d'abord du cas de la France où, quelques jours après l'accident, à la stupéfaction des pays voisins, les responsables politiques, relayés par des « experts », affirmaient sans l'ombre d'un doute à la population que le « nuage » radioactif avait contourné, et donc évité le territoire national. Cette proposition est aujourd'hui socialement traduite par : "Le nuage s'est arrêté aux frontières » et plus implicitement par: "Les autorités nous ont affirmé que la frontière avait arrêté le nuage », dans une réactivation de la mémoire historique de la Ligne Maginot. Ceci pourrait être interprété comme la découverte collective de ce que l'existence des très anciennes frontières politiques, qui furent l'un des enjeux des conflits terribles du XXème siècle en Europe, n'est plus aujourd'hui en mesure de mettre le territoire national à l'abri des périls épidémiques. La crise de la vache folle est venue renforcer, comme

\footnotetext{
${ }^{14}$ Yarochinskaya, Tchernobyl..., op. cit. p. 115.

${ }^{15}$ Carte en annexe.

${ }^{16}$ Documentaire de $52 \mathrm{mn}$, diffusé en 1999 sur la chaîne câblée Planète.

${ }^{17}$ La première réserve radio-écologique, de Polesskiy, située à quelques kilomètres de la centrale, en partie dans la zone interdite, a été inaugurée en 1988.
} 
nous l'avons déjà analysé ${ }^{18}$, ce sentiment de vulnérabilité. Il faudrait ensuite s'interroger sur les raisons pour lesquelles nous avons, plus ou moins consciemment, baptisé «nuage » une émanation gazeuse radioactive constituée de fumée mortelle plus que de vapeur d'eau. Il est vrai que la carte de la contamination est liée, pour l'essentiel, aux facteurs météorologiques des jours qui suivirent l'accident : les vents dominants poussant le «nuage » et la pluie fixant la contamination, mais la question est ailleurs. Certains scientifiques que nous avons interrogés à ce sujet en Biélorussie exprimaient clairement l'ampleur de la contamination et l'effondrement des frontières qui en résulte, telle cette femme médecin qui se référait aux fameuses photos enregistrées par le satellite Spot. Le dispositif de zonage apparaît dès lors comme un moyen de conjuration symbolique du mal et d'enfermement de la contamination alors qu'il suffit de faire varier sensiblement l'échelle de mesure surfacique de la contamination pour augmenter ou réduire l'étendue de la zone contaminée.

La catastrophe de Tchernobyl n'est donc pas un banal accident mais porte bien la marque d'une véritable catastrophe au sens où les représentations sociales de l'espace et du temps modernes s'y sont abîmées. Il semblerait que nous soyons là confrontés à l'émergence d'un monde nouveau. La Renaissance, comme toute époque, a été marquée par l'avènement d'un monde nouveau, sous le sceau de l'expansion économique et géographique, la découverte du Nouveau Monde grâce à l'usage simultané de la carte et de la boussole ; celui qui apparaît avec Tchernobyl inaugure une autre époque, celle du rétrécissement du monde habitable, un monde en régression, façonné par les conséquences du "progrès » technique, d'abord par le nucléaire et depuis peu par la génétique et les manipulations du vivant. Le monde nouveau en préparation, mais déjà en train d'advenir, est en partie produit par l'effondrement des anciennes garanties apportées par la science, et que nous avons appelée technoscience, pour son opérationnalité. Contrairement à ce qui a été longtemps affirmé, Tchernobyl n'est pas un accident (accidere, qui survient) : d'abord parce qu'il s'agit, par ses proportions et ses conséquences, d'une véritable catastrophe.

Les physiciens R. et B. Belbéoch avaient déjà constaté, en 1993, combien la gestion de la catastrophe, pour des populations, et peut-être plus encore pour les politiques et les scientifiques, révélait les difficultés conceptuelles que cet inédit posait aux représentations habituelles de l'espace, du proche et du lointain, du dedans et du dehors. Trois points au moins, selon eux, mettaient nos conceptions à l'épreuve de la nouvelle réalité, points que nous nous proposons de reprendre de manière synthétique. Notons que le lieu où se révèlent ces contradictions, c'est d'abord le langage. Ainsi avaient-ils remarqué que le recours au mot «sarcophage » pour désigner la construction de béton armé censée contenir les restes du réacteur pour une longue durée ne convenait pas à la réalité. « Sarcophage » désignait chez les Anciens la pierre dont on recouvrait le corps afin qu'il se dégrade lentement, littéralement « qu'il mange la chair », qu'il consomme le mort. Le "sarcophage » de Tchernobyl quant à lui, continue à se désagréger, et le «mort » ne se décompose pas; ce qui fait dire aux Belbéoch que «c'est finalement le mort qui mange le sarcophage ${ }^{19}$. Plus anthropologiquement, nous

\footnotetext{
${ }^{18}$ Lemarchand F., «Vaches folles, hommes fous? », Mana 4, l' sein. 1998, Université de Caen.

${ }^{19}$ Tchernobyl, une catastrophe, op. cit.
} 
pourrions conclure à l'obsolescence de la vieille culture de l'épidémie, fondée sur la logique du confinement, qui a conduit les techniciens à penser pouvoir se débarrasser d'un cadavre bien encombrant en lui créant une sépulture, cadavre dont ils connaissaient, théoriquement au moins, la «durée de vie ». Dans le même ordre d'idée, les autorités ukrainiennes ont lancé un appel d'offres en direction des pays occidentaux pour la construction d'un « abri », destiné à contenir le « sarcophage » et le réacteur, abri dont le coût était évalué entre 600 millions et un milliard de francs, selon la solution retenue. Désormais, notent les Belbéoch, " c'est à l'extérieur de "l'abri » que les gens espèrent être protégés ». Là encore, le jeu des limites et des frontières, dans une vulnérabilité nouvelle, met notre culture ancestrale du « risque », via le langage, à rude épreuve. Enfin, face à la menace de contamination de la mer Noire dans la mesure où la radioactivité accumulée sur le site de Tchernobyl contamine les nappes souterraines et, surtout, la rivière Dniepr qui alimente le bassin réservoir de la ville de $\mathrm{Kiev}^{20}$, on avait, dès 1986 , construit des digues et un mur souterrains pour empêcher les eaux en provenance de la zone proche du réacteur, d'atteindre la rivière Pripyat, affluent du Dniepr. Une centaine de barrages filtrants ont ainsi été construits, barrages qui ont d'ailleurs montré leur vulnérabilité en période de crues, au cours desquelles les radionucléides débordent et se redéposent en aval. Bâtir, pour le moins paradoxalement, des digues en sous-sol pour protéger les rivières n'a pas, une fois encore, prouvé l'efficience de nos connaissances en pareille situation. Ainsi, concluent les Belbéoch, "la mer Noire où se jette le Dniepr n'a guère de chance d'échapper à la contamination $\|^{21}$.

Bien que la catastrophe nucléaire recèle encore, et probablement pour longtemps, une large part d'impensé, nous nous risquerons à avancer quelques hypothèses au sujet de ce qui nous est apparu comme une forme nouvelle de déracinement, qui ressortit des contradictions et des paradoxes que nous avons évoqués au sujet des Samossiols. On peut, pour la caractériser, parler d'un déracinement sans déplacement, in situ, immobile, d'un d'arrachement de l'intérieur, incommensurable aux formes qu'il avait prises jusqu'alors, formes organisées de l'exode, paysans chassés de leurs terres au Brésil, et plus largement par toutes les conséquences de l'urbanisation et de l'industrialisation des sociétés modernes, puis des pays du «sud». Avec Tchernobyl, les dimensions du temps et de l'espace, dans l'intimité de leur articulation, ont été bouleversées et il est devenu impossible de penser le rapport à l'espace sans prendre en compte également, comme nous allons le voir, la dimension temporelle de la catastrophe. Au-delà des renversements opérés entre le dedans et le dehors, dans la complexe problématique de la «zone » qui ne correspond à aucune mémoire, à aucune expérience politique, ou encore entre le proche et le lointain, dans les jeux tout aussi complexes de révélation de la vulnérabilité de territoires à l'échelle planétaire, il faudra encore longuement s'interroger sur l'expérience faite par quelque six millions de personnes dont le territoire dans lequel elles vivent apparemment « comme avant » leur est en fait devenu étranger. Le monde, négatif, contaminé, fabriqué par la technoscience n'est plus désormais, comme nous nous sommes efforcés de le montrer, le monde d'avant. Il faudra également s'interroger sur le prix de la - supposée adaptation des

\footnotetext{
${ }^{20}$ Le Ministère des situations extrêmes admet que les eaux du Dniepr qui alimentent en eau potable plus de 32 millions de personnes en Ukraine « ont été considérablement contaminées ».

${ }^{21}$ Ibid. p. 165.
} 
hommes dans un territoire durablement contaminé et devenu dangereux pour la vie elle-même. On ne peut en effet, comme nous l'avons expérimenté, goûter un fruit de cette terre sans être saisi d'un doute, sans lui trouver cet arrière-goût d'étrangeté, immédiatement refoulé et enfoui au plus profond de l'inconscient, qui ne laisse plus affleurer qu'un sentiment d'anxiété, d'angoisse diffuse. On ne peut non plus contempler un paysage rural, répondant pourtant aux critères de l'esthétique romantique occidentale par ses forêts, ses rivières, sa palette de couleurs, ses jeux d'ombres et de lumières, l'omniprésence d'animaux rustiques et sauvages etc., sans être saisi par l'effroi à l'idée qu'en dépit des apparences, il s'agit bien du monde nouveau, produit de la catastrophe nucléaire, le « monde d'après l'apocalypse » décrit dans son horreur par Svetlana Alexiévitch $^{22}$. Dans une certaine mesure, la redéfinition de la dimension spatiale du territoire pourrait être interrogée à la lumière de la construction d'une identité négative des habitants des zones contaminées par ceux qui croient ou prétendent ne pas y habiter. S'il est vrai qu'avec le temps le phénomène de rejet appelé «syndrome de Tchernobyl », illustrant la stigmatisation des «Tchernobyliens », s'est estompé, il n'en demeure pas moins que le statut des habitants de la « zone » reste ambivalent.

\section{La catastrophe technologique ou le temps rompu.}

Nous pourrions émettre l'hypothèse que l'époque qui s'inaugure pourrait être pensée à partir d'une réflexion sur les catastrophes engendrées par l'homme et en particulier, pour ce qui concerne notre propos, sur les catastrophes technologiques. L'apparition de la modernité a coïncidé avec la fin d'une conception religieuse de la catastrophe et avec l'émergence d'un imaginaire catastrophique nouveau qui se révélait comme unique moyen d'appréhender le monde, alors en train d'échapper à toute compréhension. A son tour, la nécessité de penser les dessins d'une nouvelle époque caractérisée en partie au moins par le développement technoscientifique, et qui semble de nouveau échapper à toute compréhension, devrait nous amener à reconsidérer l'imaginaire catastrophique moderne à partir duquel nous pensons notre rapport au monde depuis le XVIIIème siècle. Quelque chose a changé dans la nature même de la catastrophe à partir du moment où elle s'est trouvée liée au développement conjoint de la science et de la technique. Dans le cas de Tchernobyl, il convient de prêter la plus grande attention, contrairement aux medias qui ont escamoté la réalité aussi rapidement qu'ils l'ont faite apparaître, à la durée des conséquences, et donc de séparer la phase accidentelle à proprement parler, de l'ampleur catastrophique qu'a prise l'événement. Le développement généralisé d'une pollution à l'échelle planétaire, les surfaces contaminées par la radioactivité $(6,4$ milliards de Curies rejetés, les seuls rejets en césium-137 équivalent à 300 Hiroshima, 7220000 ha de terres agricoles contaminés), entraînent une production permanente des faits catastrophiques. Les effets, quotidiens et durables, deviennent alors incommensurables aux conséquences de n'importe quelle catastrophe naturelle dans la mesure où tout espoir de réparation semble abandonné, ou trop lointain pour être représentable. Loin de s'améliorer, la situation - c'est-àdire principalement la santé des populations - n'a cessé d'empirer, de même que l'état général

\footnotetext{
${ }^{22}$ Alexiévitch S., La Supplication, op. cit.
} 
de l'environnement des pays industrialisés. La réalisation de la catastrophe semble avoir porté atteinte à notre possibilité même de la figurer, comme le remarque A. Lebrun ${ }^{23}$ : «plus la catastrophe est plausible ou réelle, moins elle est imaginée ». Ainsi, poursuit-elle, dans les innombrables fictions cinématographiques ou littéraires liées à la bombe nucléaire, "la fin du monde n'est plus figurée alors même que nous disposons pour la première fois des moyens de la provoquer ». La peur que nous pouvons éprouver à l'égard de la nature n'est plus liée désormais à ses manifestations spontanées et dévastatrices, mais, comme l'a montré H. Jonas ${ }^{24}$, au mépris de son équilibre et aux effets des actions - aux conséquences à présent irréversibles - de l'homme. Au surgissement de la situation nucléaire comme réalité répond un refoulement généralisé du danger d'anéantissement général devenu réel. En ce sens, c'est bien le pouvoir critique de la catastrophe imaginaire comme imaginaire de la catastrophe qui disparaît, au profit de la mise en oeuvre de dispositifs gestionnaires et experts des effets de la catastrophe réelle.

La catastrophe devient, dans les sociétés modernes, selon Henry-Pierre Jeudy ${ }^{25}$, un moyen d'adaptation, et donc de socialisation, des individus à leur nouvelle condition humaine, une condition à haut risque, et il se déploie, dans les sociétés industrielles, une certaine idée qu'il nous faudra désormais «faire avec » en produisant les efforts physiques et psychiques nécessaires pour s'adapter... ou disparaître. Cela explique, dans le cas de Tchernobyl, que l'on puisse encore admettre le fonctionnement - dans des conditions des plus précaires - de la centrale, via un bricolage technique, comme si rien ne s'était passé. L'imaginaire catastrophique vient alors redoubler le réel au lieu de nous inciter à le changer. Exit les utopies radieuses du XVIIIème, les grandes utopies de Ledoux ou de Montesquieu, l'imaginaire catastrophique ${ }^{26}$ semble désormais décrire, depuis une dizaine d'années, des reprises de la vie, en situation postcatastrophiques, qui ont pour principale caractéristique d'être ponctuelles et étrangères à tout équilibre naturel, comme des «bulles de nature ». Les projets « hors-sol» liés à la création d'univers de synthèse - que les Etats-Unis ont d'ailleurs tenté d'expérimenter dans le projet Biosphère $\mathrm{II}^{27}$ - consacrent le rôle de la technique dans la production contemporaine d'artifices du vivant, de mondes clos sur eux-mêmes, indifférents les uns aux autres, autonomes et autogérés. Dans cette logique de résignation, ces nouvelles architectures hors sol, ces prothèses, ne modifient jamais le monde dévasté où elles s'insèrent, pas plus qu'elles ne sont modifiées par lui. La supposée nécessité de s'adapter ne peut, dans cet imaginaire, que recourir d'une manière sans cesse grandissante à l'artifice technique, pour répondre à un problème supposé d'ordre technique.

L'analyse des croyances et des pratiques mises en oeuvre par les habitants des territoires contaminés nous a permis de montrer que, pour l'essentiel, ces pratiques et croyances

\footnotetext{
${ }^{23}$ La Perspective dépravée, Bruxelles, La lettre volée, 1991.

${ }^{24}$ H. Jonas Le Principe responsabilité, Paris, Ed. du Cerf, 1990.

${ }^{25}$ Voir Le Désir de catastrophe, Paris, Aubier, 1990.

${ }^{26}$ Nous faisons d'abord référence à la littérature et au cinéma de science-fiction des années quatre-vingt, mais également à la manière dont est envisagée la catastrophe par les « gestionnaires » du risque.

${ }^{27}$ Il s'agissait, aux confins d'un désert, de recréer artificiellement et de faire coexister différents biotopes représentatifs de la diversité biologique terrestre, enfermés dans une gigantesque bulle. Le coûteux projet se solda par un cuisant échec.
} 
visent à déplacer le lieu où l'on doit affronter la réalité - l'objectivité de la situation à risque - dans le registre de l'imaginaire et du symbolique. Il ne s'agit, certes, pas d'une nouveauté dans la mesure où, tant que les catastrophes ont été exclusivement naturelles, l'imaginaire catastrophique s'est déployé dans l'univers mystique et religieux ; au pire, la catastrophe était considérée comme faisant partie du quotidien, de l'ordre des choses. Or, on assiste avec les catastrophes technologiques contemporaines, à de nouvelles formes d'expiation du malheur que nous appellerons dénis de réalité. Ce refus de réalité ne trouvant pas d'alternative symbolique qui autorise à parler la peur, vient alors à manquer une explication au malheur qui permette de lui donner un sens. Au bout du compte et bien que les attitudes face au risque soient, comme nous l'avons analysé, souvent paradoxales, nombreuses sont les réactions des autochtones qui nient totalement l'existence d'un risque lié à la contamination et prétendent échapper ainsi à l'emprise de la menace. On pourrait également faire état, que l'on soit en situation postcatastrophique (Tchernobyl), comme en situation pré-catastrophique (la situation d'alerte permanente que connaissent les pays industrialisés), de l'existence de nombreux rites conjuratoires censés minimiser, voire évacuer le risque nucléaire. C'est ce que doit mettre à jour l'analyse des rumeurs: la théorie de "l'innocuité des faibles doses », ou l'idée qu'une accoutumance aux radiations est possible par exemple, participent de ces productions sociales qui permettent d'accepter une situation que, seules, ni la raison, ni les cultures anciennes ne semblent pouvoir « rationaliser ».

Lors, la durabilité des effets et des conséquences délétères pour la santé de manifestations de la réversibilité du développement technoscientifique, ne nous autorise plus à faire entrer la catastrophe dans le domaine du passé, ni en l'inscrivant dans un récit, une narration historique, ni en la saisissant par le biais d'une mémoire collective qui permettrait, par sa puissance d'oubli actif, de faciliter le travail de deuil et de dépasser la catastrophe. Pour S. Alexiévitch, la difficulté à prendre la véritable mesure de la catastrophe in situ tient au fait que deux catastrophes ont coïncidé : "L'une sociale sous nos yeux, un immense continent socialiste a fait naufrage l'autre, cosmique - Tchernobyl. Deux explosions totales. Mais la première est plus proche, plus compréhensible. Les gens sont préoccupés par le quotidien: où trouver l'argent pour vivre? Où aller? Que croire? Sous quelle bannière se ranger? Chacun vit cela. Mais tous voudraient oublier Tchernobyl. Au début, on espérait le vaincre, mais comprenant la vanité de ces tentatives, on se tut. Il est difficile de se protéger de quelque chose que nous ne connaissons pas, que l'humanité ne connaît pas. Tchernobyl nous a transposés d'une époque dans une autre ${ }^{28}$. L'un des principaux facteurs sur lequel repose l'hypothèse selon laquelle on ne pourrait pas appréhender la catastrophe de Tchernobyl comme n'importe quel autre avatar technologique est donc constitué du cadre social-historique et idéologique dans lequel s'inscrit l'événement, et en particulier des transformations qu'il a subies depuis la survenue de l'accident, il y a treize ans. Il s'agit, en d'autres termes, de comprendre ce qui, dans l'effondrement du « bloc de l'est » en tant que ce dernier constituait l'une des principales formes politico-économiques de société moderne, ne permet plus d'inscrire la catastrophe de Tchernobyl dans une mémoire

\footnotetext{
${ }^{28}$ Alexiévitch S., La Supplication, op. cit. p. 34.
} 
sociale $^{29}$ et historique. J. Chesneaux notait à ce sujet : "La catastrophe de Tchernobyl a cassé net à la fois le discours d'infaillibilité de la technoscience nucléaire et le discours de légitimité de l'ordre soviétique. Tout, y compris le consensus résigné dont l'un et l'autre bénéficiaient, $s^{\prime}$ est trouvé soudain remis en cause ${ }^{30}$. Rappelons que les événements majeurs de la modernité occidentale ont tous dû, par le passé, s'inscrire dans le cours d'une histoire générale. L'histoire moderne a pu ainsi fonctionner, depuis le XVIIIème siècle, comme cadre général d'un « grand récit », comme cadre pour une multitude de petits récits particularistes, individuels, familiaux ou communautaires. Ainsi, chacune des histoires qui se tissent dans les rapports sociaux directs entre les hommes, dans le registre de la socialité primaire ${ }^{31}$, tend à inscrire son sens dans un projet global et universaliste qui contribue à lui donner un sens. Si chacune de nos histoires singulières n'épuise pas son sens dans le cadre de cette histoire moderne universelle, cette dernière fournit en revanche un support commun susceptible de recevoir une grande diversité de récits produits de corporations, de communautés ou d'individualités. Qu'il s'agisse d'histoires de mineurs désoeuvrés, de paysans liquidés, d'anciens combattants ou de rescapés de la déportation, la rationalisation du malheur supporté par chacun avait jusqu'à présent, du moins l'a-t-on pensé, trouvé son sens par la mobilisation d'une histoire moderne universaliste qui permettait de légitimer, au nom des valeurs du droit universel, le sacrifice de ceux qui ont donné leur vie ou leur santé physique et psychique au nom des "intérêts supérieurs de la nation ». L'histoire des sociétés occidentales a engendré, au cours du XXème siècle, des communautés de vaincus, vaincus de la technique (des militaires et des victimes civiles de la Grande Guerre, à ceux et celles de la guerre du Golfe ou du Kossovo) et du productivisme (les communautés et classes désoeuvrées issues du projet productiviste : ouvriers et paysans). Il faudrait dès lors s'interroger sur le statut des victimes, des «vaincus » de la catastrophe de Tchernobyl, et en premier lieu des 800000 liquidateurs mobilisés. L'effort qui a été demandé aux populations des pays concernés de travailler dans ou à proximité des sites industriels nucléaires, en en acceptant les risques inhérents au nom d'un grand projet national, et, à présent, l'effort qui est demandé à ces mêmes populations de vivre dans des territoires contaminés avec les conséquences que cela implique, trouveront-ils une légitimité «historique»? De quelle histoire sera faite cette histoire-là s'interrogeait Svetlana Alexiévitch ? Günter Anders remarquait, dans son essai de définition de l'humanité à partir de notre existence « sous le signe de la bombe atomique », que certains événements ne sont plus des catastrophes historiques, que, ne passant plus par le crible de l'histoire, ils constituent une fin de l'historicité, des «blocs saillants de l'histoire »: "Il en va de même, pensait-il, des essais nucléaires, sans parler des guerres atomiques. Il se peut que leurs préparatifs aient encore une dimension historique (...) mais dès l'instant où il y aurait un passage à l'acte, c'en serait fini de l'histoire. Ce qui subsisterait ne serait plus une situation historique mais un champ de ruine sous lequel serait enfoui tout ce qui a été un jour, l'histoire. Et si l'homme malgré tout survivait, ce ne serait plus un être historique mais un résidu pitoyable : un élément pollué dans une nature polluée $»^{32}$. Si le sort dramatique des liquidateurs

\footnotetext{
${ }^{29}$ Nous reprenons l'opposition instaurée par le sociologue M. Halbwachs entre mémoire sociale ou historique, officielle, et mémoire collective, communautaire ou familiale. Cette dernière ne fonctionne qu'à partir de cadres sociaux (des personnes, des objets, des lieux) qui lui servent de support et elle se joue de la vérité historique.

${ }^{30}$ Chesneaux J., Habiter le temps, op. cit. p. 276.

${ }^{31}$ A Caillé, Splendeurs et misères des sciences sociales, La Découverte, Paris, 1988.

${ }^{32}$ Anders G., De la bombe..., op. cit. p. 46.
} 
a conduit les nations concernées à reconnaître leur courage et leur bravoure à l'instar des héros des nations modernes, il est assez probable qu'on ne rende jamais hommage aux quelques millions de personnes qui auront expérimenté la première catastrophe technologique de l'humanité : "Ils étaient venus d'Erevan jusqu'à Tchernobyl, écrit Alla Yarochinskaya, pour se retrouver dans la fournaise, en enfer, et y oeuvrer jusqu'au péril de leur vie ! Et l'État, après les avoirs utilisés, les avait aussitôt oubliés (...) même les enfants présentant des affections de la thyrö̈de ne sont pas suivis en tant que victimes de la catastrophe! " ${ }^{33}$. Non seulement aucun musée, aucun monument, aucun récit, pour l'instant, n'est là pour rendre compte de cette tragédie à une échelle universelle, mais l'identité même des vrais liquidateurs fut largement usurpée par ceux qui, occupant des postes administratifs, trouvèrent là un moyen d'obtenir quelques avantages sociaux.

Les modes de distribution de la mémoire que sont le monumental et le muséal peuventils d'ailleurs prétendre remplir une telle tâche? Pourra-t-on, comme Alla Yarochinskaya, affirmer un jour : "Ce village est un monument, un monument à Tchernobyl. Un monument au crime $»^{34}$. La politique d'effacement des traces, d'enterrement des villages et de mise entre parenthèses de leur nom sur les cartes, engagée depuis 1997, semble indiquer que non, et si la «zone» devient temple ou musée, c'est plus probablement sous la forme en devenir d'une réserve de «nature ». Les territoires sinistrés apparaissent ainsi comme des lieux où se conjuguent de multiples figures de la ruine, politique, sociale, environnementale, économique...

Le monde, le territoire des habitants des zones contaminées, s'est, pour la plupart d'entre eux, refermé, étiolé, resserré jusqu'aux frontières de l'intimité. Repliés sur leur village, leur lopin ou leur jardin, les Tchernobyliens ne vivent plus que dans et par la «zone». Les problèmes sont locaux et spécifiques, et il semble inconcevable de produire un effort permanent pour «s'adapter » à cette vie nouvelle tout en imaginant qu'une autre vie, «normale », serait possible ailleurs. Du point de vue de la construction de la responsabilité, Roger et Bella Belbéoch avaient remarqué que l'«on exigeait le jugement des coupables » mais qu'en fait « c'étaient les responsables de la gestion post-accidentelle qui étaient principalement visés et non les responsables de la catastrophe, concepteurs et exploitants de la centrale ${ }^{35}$, comme si l'on ne pouvait pas atteindre le lieu et le temps de l'origine de ce qui a littéralement bouleversé le monde, la catastrophe. Si un hypothétique horizon d'attente peut encore s'ouvrir à ces populations, "des gens perdus, occultés, trompés, comme des personnes âgées qui n'attendent plus rien ${ }^{36}{ }^{3}$, c'est probablement à une échelle particulariste et en puisant largement dans les formes sociales et modes de production issus de l'époque prémodeme qu'elles ont su garder en mémoire.

\footnotetext{
${ }^{33}$ Yarochinskaya A., Tchernobyl, vérité interdite, op. cit. p. 69.

${ }^{34}$ Ibid. p. 34.

${ }^{35}$ Belbéoch R. et B., Tchernobyl, une catastrophe, op. cit., p. 181.

${ }^{36}$ Yarochinskaya A., Tchernobyl, vérité isnterdite, p. 21.
} 